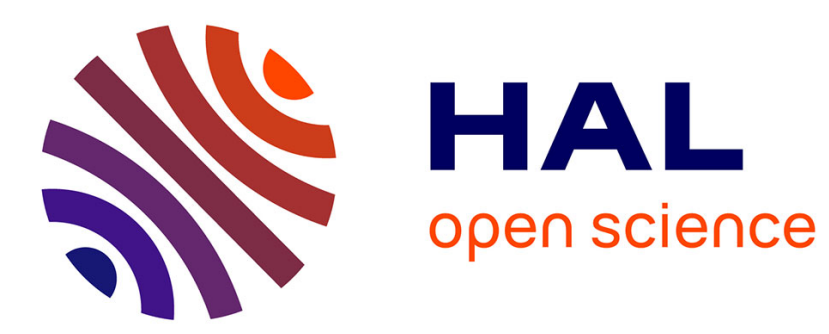

\title{
Effects of mutations and glycosylations on STS activity: A site-directed mutagenesis study
}

Chloe Stengel, Simon P. Newman, Joanna M. Day, Helena J. Tutill, Michael J. Reed, Atul Purohit

\section{- To cite this version:}

Chloe Stengel, Simon P. Newman, Joanna M. Day, Helena J. Tutill, Michael J. Reed, et al.. Effects of mutations and glycosylations on STS activity: A site-directed mutagenesis study. Molecular and Cellular Endocrinology, 2008, 283 (1-2), pp.76. 10.1016/j.mce.2007.11.012 . hal-00531963

\section{HAL Id: hal-00531963 https://hal.science/hal-00531963}

Submitted on 4 Nov 2010

HAL is a multi-disciplinary open access archive for the deposit and dissemination of scientific research documents, whether they are published or not. The documents may come from teaching and research institutions in France or abroad, or from public or private research centers.
L'archive ouverte pluridisciplinaire HAL, est destinée au dépôt et à la diffusion de documents scientifiques de niveau recherche, publiés ou non, émanant des établissements d'enseignement et de recherche français ou étrangers, des laboratoires publics ou privés. 


\section{Accepted Manuscript}

Title: Effects of mutations and glycosylations on STS activity:

A site-directed mutagenesis study

Authors: Chloe Stengel, Simon P. Newman, Joanna M. Day, Helena J. Tutill, Michael J. Reed, Atul Purohit

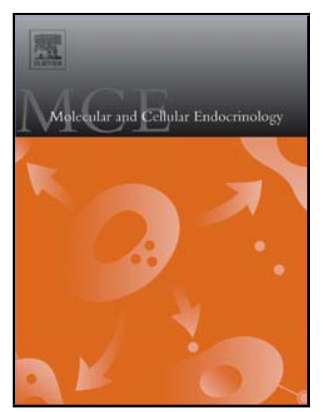

PII:

S0303-7207(07)00430-3

DOI: doi:10.1016/j.mce.2007.11.012

Reference: MCE 6757

To appear in: Molecular and Cellular Endocrinology

Received date: 19-10-2007

Revised date: 19-11-2007

Accepted date: $19-11-2007$

Please cite this article as: Stengel, C., Newman, S.P., Day, J.M., Tutill, H.J., Reed, M.J., Purohit, A., Effects of mutations and glycosylations on STS activity: A site-directed mutagenesis study, Molecular and Cellular Endocrinology (2007), doi:10.1016/j.mce.2007.11.012

This is a PDF file of an unedited manuscript that has been accepted for publication. As a service to our customers we are providing this early version of the manuscript. The manuscript will undergo copyediting, typesetting, and review of the resulting proof before it is published in its final form. Please note that during the production process errors may be discovered which could affect the content, and all legal disclaimers that apply to the journal pertain. 
Effects of mutations and glycosylations on STS activity: a site-directed mutagenesis study Chloe Stengel $^{1}$, Simon P. Newman ${ }^{1}$, Joanna M. Day ${ }^{1}$, Helena J. Tutill ${ }^{1}$, Michael J. Reed ${ }^{1}$ and Atul Purohit ${ }^{1}$

${ }^{1}$ Endocrinology and Metabolic Medicine and Sterix Ltd, Faculty of Medicine, Imperial College London, St Mary's Hospital, London, W2 1NY, U.K.

Short title: STS: structure and activity

Proofs and correspondence to:

Chloe Stengel

Endocrinology and Metabolic Medicine

Second Floor, Mint Wing

Faculty of Medicine, Imperial College London

St Mary's Hospital

Mint Wing, Second Floor

London

W2 1NY, U.K.

Tel: (+) 442078861210

Fax: (+) 442078861790

Email: c.stengel@imperial.ac.uk

\section{Funding}

This research was supported by Sterix Ltd., a member of the Ipsen Group. 


\begin{abstract}
Steroid sulphatase (STS) catalyses the formation of active steroids from inactive steroid sulphates. High levels of intra-tumoural STS mRNA are associated with a poor prognosis in postmenopausal patients with oestrogen receptor positive breast cancer. In this study, analysis of the mutated STS protein showed that N- and C-terminal truncated STS constructs are inactive. Histidine 136, located inside the active site, is crucial for STS activity whereas proline 212, which allows the protein turn into the membrane, is not. Mutations in glycosylation sites asparagine 47 and 259 decreased STS activity while asparagine 333 and 459 mutations did not affect it. However, immunoblot studies revealed that all four N-linked sites are glycosylated to some extent. In addition, a polyclonal antibody raised in rabbit against human STS was developed and characterised. These data increase our knowledge of the STS enzyme structure and may help design new STS inhibitors.
\end{abstract}

Keywords: Steroid sulphatase, mutagenesis, glycosylation, STS antibody, breast cancer, MCF-7 cells. 


\section{Introduction}

Human steroid sulphatase (STS, EC 3.1.6.2) contributes to the maintenance of high levels of the active oestrogen, 17 $\beta$-oestradiol, in breast tumour cells. STS is present in breast carcinomas and in oestrogen receptor (ER) dependent breast cancer cells (Billich et al., 2000; Pasqualini et al., 2001). STS mRNA expression is a predictor of recurrence in breast cancer patients (Utsumi et al., 1999), and increased STS expression in ER positive tumours is correlated with a poor prognosis (Miyoshi et al., 2003). STS gene deletions and point mutations in the STS C-terminal domain are associated with X-linked ichthyosis (XLI) (Alperin and Shapiro, 1997).

The STS enzyme is localized in the endoplasmic reticulum, which catalyses the desulphation of inactive cholesterol sulphate and other 3-hydroxysteroid sulphates, such as pregnenolone sulphate (PREG S), dehydroepiandrosterone sulphate (DHEAS) and oestrone sulphate (E1S), to their unconjugated active forms, cholesterol, pregnenolone, DHEA and oestrone respectively (Reed et al., 2005). STS is ubiquitously expressed in mammalian tissues (Warren and French, 1965). The STS gene has been cloned, characterized (Yen et al., 1987) (GenBank accession no. $\underline{\mathbf{M 1 6 5 0 5}}$ and $\underline{\mathbf{J 0 4 9 6 4}}$ ) and its functional structure has been studied (Hernandez-Guzman et al., 2003). The encoded protein is synthesized as a membrane-associated precursor with an apparent size of $63 \mathrm{kDa}$, upon cleavage of the signal peptide the size of STS decreases to $61 \mathrm{kDa}$ (Conary et al., 1986). The STS protein contains four possible N-linked glycosylation sites (Stein et al., 1989; Yen et al., 1987). The N-glycosylation sites are indicated by the consensus sequence AsnXaa-Ser/Thr. However, the presence of this sequence does not mean that the site is always glycosylated. Digestion of STS by endoglycosidase H shows that at least two (Asn47 and Asn259) of the STS N-glycosylation sites are used for glycosylation. It has also been reported that although both the N-terminal region and the C-terminal region of the STS enzyme are important for the STS enzymatic function (Sugawara et al., 2006), the key catalytic residue in sulphatase is a unique $\mathrm{C} \alpha$-formylglycine, which is generated from a cysteine precursor. The $\mathrm{C} \alpha$ formylglycine functions as a nucleophilic aldehyde hydrate in the initial addition reaction of sulphate ester hydrolysis (von Figura et al., 1998).

Inactivation of the STS gene results in X-linked ichthyosis (XLI), one of the most prevalent human inherited errors of metabolism (Shapiro et al., 1989). In 80-90\% of cases, the XLI is due to complete deletion of the $146 \mathrm{~kb}$ STS gene and substantial flanking regions from the distal 
short arm of the X chromosome. In a minority of XLI patients, mutations in the STS gene have been reported to inactivate the enzyme (Alperin and Shapiro, 1997). The study of these patients led to the identification of several point mutations, all of which catalytically inactivate STS (Basler et al., 1992; Gonzalez-Huerta et al., 2003; Sugawara et al., 2000; Valdes-Flores et al., 2001).

The key role that the STS enzyme plays in regulating the formation of active steroids means that the enzyme represents an inhibitory target for the treatment of hormone-dependent breast cancer. Oestrone-3-O-sulphamate was identified as the first irreversible STS inhibitor (Purohit et al., $1995 b)$ but proved to be oestrogenic in rats (Elger et al., 1995). However, other selective sulphatase inhibitors showed promise as antiproliferative agents in hormone-dependent breast carcinoma (Purohit et al., 1999) and the recent phase I clinical studies testing STX64, a tricyclic coumarin sulphamate, in female breast cancer patients have shown very encouraging results (Stanway et al., 2006).

In this study, we analysed the activity of mutated forms of STS to investigate and define the regions of the enzyme that are important for its activity. Furthermore, the role of all four potential glycosylation sites in modulating the activity of the STS enzyme was explored. 


\section{Materials and methods}

\section{Cell culture}

293-EBNA cells (Invitrogen, Paisley, United Kingdom) and JEG-3 cells (American Type Culture Collection, LGC Promochem, Teddington, United Kingdom) were cultured in Dulbecco's Modified Essential medium supplemented with 10 \% (v/v) fetal calf serum, $1 \%$ Lglutamine $(200 \mathrm{mM}), 1 \%$ sodium bicarbonate $(7.5 \%)$ and $250 \mu \mathrm{g} / \mathrm{ml}$ geneticin sulphate (G418, Sigma, Poole, United Kingdom). Monkey kidney COS-1 cells and ER positive human breast cancer MCF-7 cells were obtained from ATCC and routinely cultured in RPMI 1640 medium supplemented with $10 \%$ (v/v) fetal calf serum, $1 \%$ L-glutamine $(200 \mathrm{mM}), 1 \%$ Non-essential amino acids $(100 \mathrm{X})$ and $1 \%$ bicarbonate $(7.5 \%)$ from Sigma. Cells were maintained in humidified $5 \% \mathrm{CO}_{2}$ atmosphere at $37^{\circ} \mathrm{C}$.

\section{Expression of His $_{6}$-tagged STS}

To aid purification of the STS protein, codons coding for six histidines (His ${ }_{6}$ tag) were added to the 3' end of wild type human STS cDNA by PCR, immediately prior to the TAG stop codon. The tagged STS cDNA was subcloned into pCEP4, transfected into 293-EBNA cells, and a cell line with stable over-expression of STS-His $_{6}, 293$-EBNA[His 6 STS], was derived by selection with $250 \mu \mathrm{g} / \mathrm{ml}$ Hygromycin B (Sigma) over several passages. The STS cDNA was kindly provided by Dr Stein and Dr Shapiro (Shapiro et al., 1989; Stein et al., 1989).

\section{Purification of $\mathrm{Hi}_{6}$-tagged STS}

293-EBNA[his 6 STS] cells were grown to confluency in batches of $10 \times$ T-150 poly-D-lysinecoated flasks (Biocoat, BD Biosciences, Cowley, United Kingdom). After PBS washing, the cells were solubilised in Buffer A (0.5 \% Triton-X in $250 \mathrm{mM}$ sucrose / PBS buffer with $10 \%$ 
glycerol and $1 \mathrm{mM}$ PMSF). His 6 -tagged STS protein was purified by affinity chromatography using $\mathrm{NiCl}_{2}$-primed HisTrap Chelating columns (GE Healthcare, Amersham, United Kingdom) and eluted with $500 \mathrm{mM}$ imidazole in Buffer A. The purified protein (1.5 mg) was concentrated approximately 6-fold using ICON concentrators (Pierce, Rockford, USA), aliquotted and stored at $-80^{\circ} \mathrm{C}$.

\section{Production and characterisation of STS antisera}

Antibodies were raised in mice (x3) and rabbits (x2) according to a standard protocol (one immunisation plus 3 boosts; Eurogentec, Seraing, Belgium). Antisera titre was established by a 96 well plate ELISA assay (Engvall and Perlman, 1971) using $2 \mu \mathrm{g} / \mathrm{ml}$ purified STS-His 6 as antigen and either a secondary anti-rabbit or anti-mouse AP-conjugated IgG (7056 or 7054 respectively, Cell Signaling Technology, Boston, MA) at 1:2,000. A monoclonal antibody to the His $_{6}$ tag was used as a primary antibody positive control. Immunoreactivity was quantitated at 405 nm (FLUOstar OPTIMA, BMG Labtech, Aylesbury, United Kingdom) after 20 minutes of exposure to r-nitrophenyl phosphate substrate (N7653, Sigma). The specificity of the antisera for the STS moiety of STS-His 6 was confirmed by immunoblotting using several STS protein sources and other his ${ }_{6}$-tagged proteins.

\section{Preparation of the mutated STS constructs}

A plasmid encoding wild type human STS (pSTSwt) was prepared by inserting the human STS cDNA fragment $(2.4 \mathrm{~kb}$; link to [11] [18]) into pALTERMax (Promega Ltd, Southampton, United Kingdom) between $\mathrm{XbaI}$ and SmaI restriction endonuclease sites. STS cDNA mutants were generated by site-directed mutagenesis using the pALTERMax kit from Promega according to the manufacturer's instructions. A series of primers was designed to cause the desired amino acid change in the STS sequence within pALTERMax vector. Nine point mutations, pSTSN47Q, pSTS-N54Q, pSTS-H136Y, pSTS-H136W, pSTS-H136F, pSTS-P212G, pSTS-N259Q, pSTS-N333Q, pSTS-N459Q and one double mutation pSTS-N47/259Q (Fig. 1A) were 
generated. Two truncated STS expression constructs (Fig. 1B, 1C) were prepared by selective restriction enzyme digestion and cloning the truncated products back into pALTERMax vector. pSTS1-184 lacks 399 C-terminal amino acids and pSTS21-583 lacks the first $21 \mathrm{~N}$-terminal amino acids that encode the signal peptide. All mutated and truncated constructs were sequenced to ensure the correct sequence changes were present.

\section{Transfection}

COS-1 cells and MCF-7 cells were grown to $80 \%$ confluency and transfected with STS expression vectors using Amaxa nucleofection technology (Amaxa GmbH, Koeln, Germany) according to the protocol of the manufacturer. $72 \mathrm{~h}$ after transfection the STS activity was determined and the protein extracts were collected for immunoblotting.

STS enzyme activity

Cells were incubated overnight with $\left[6,7-{ }^{3} \mathrm{H}\right]$ E1S $\left(5\right.$ pmol, 7 x $10^{5} \mathrm{dpm}, 60 \mathrm{Ci} / \mathrm{mmol}$; PerkinElmer LS, Wellesley, MA). The product E1 was separated from E1S by toluene partition using $\left[4-{ }^{14} \mathrm{C}\right] \mathrm{E} 1$ to monitor procedural losses, and radioactivity was measured by liquid scintillation counting (Purohit et al., 1995a).

\section{Immunoblot analysis}

Total cellular protein was prepared with $350 \mu \mathrm{L}$ of ice cold RIPA buffer (Sigma) and $3.5 \mu \mathrm{L}$ protease-phosphatase inhibitors at $4{ }^{\circ} \mathrm{C}$ for $30 \mathrm{~min}$. The extracts were centrifuged $(15 \mathrm{~min}$ at $13000 \mathrm{rpm}$ at $4{ }^{\circ} \mathrm{C}$ ) and total protein concentrations were determined by Bradford assay with Bradford reagent (Sigma). Half of each protein extract was denatured at $100{ }^{\circ} \mathrm{C}$ in $1 \mathrm{X}$ glycoprotein denaturing buffer and deglycosylated with $10 \%$ Peptide-N-Glycosidase F in $1 \mathrm{X}$ G67 reaction buffer with $1 \% \mathrm{NP} 40$ at $37^{\circ} \mathrm{C}$ for $1 \mathrm{~h}$ (buffers and endoglycosidase purchased 
from New England Biolabs, Hitchin, United Kingdom). $20 \mu \mathrm{g}$ of each protein samples were denatured at $100{ }^{\circ} \mathrm{C}$ for 10 minutes, separated by electrophoresis using 4-12\% Bis-Tris Nupage gels (Invitrogen) and blotted onto a PVDF membrane (GE Healthcare). The membrane was treated with the blocking buffer ( $3 \%$ BSA in PBS-Tween $1 \%$ ) for $2 \mathrm{~h}$ and incubated overnight at $4{ }^{\circ} \mathrm{C}$ with an in-house anti-human STS polyclonal antibody (SK3706) at 1:250 in 1\% BSA in PBS-Tween $1 \%$. After 3 washes with PBS-Tween $1 \%$, the membrane was incubated for $1 \mathrm{~h}$ with the secondary anti-rabbit IgG Ap-linked antibody (ref. 7054, Cell Signaling Technology, Boston, MA), washed three times, developed with ECF substrate (GE Healthcare) and visualised using a Storm imaging system (GE Healthcare). 


\section{Results}

Immunoreactivity of STS antisera

Antisera titre was established by ELISA assay using purified STS-His 6 as antigen. All five antisera were immunoreactive in the ELISA assay (Fig. 2A). Antisera from the two rabbits (SK3706 and SK3707) had stronger reactivity than the control His 6 tag monoclonal mouse antibody, whereas those from all three mice (SKM12, SKM13 and SKM14) were weaker. Immunoblot analysis indicated that all antisera recognised the STS protein without recognition of

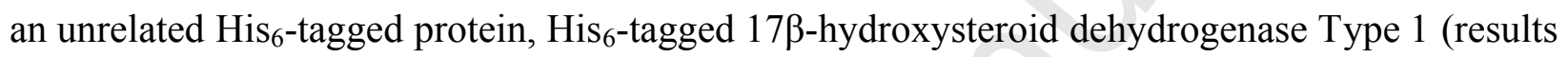
not shown), confirming specificity for the STS moiety of the STS-His 6 protein. SK3706 was optimised for use in further immunoblotting experiments, and recognised STS protein in lysates from over-expressing cell lines (293-EBNA[His ${ }_{6}$ STS], COS-1.STS), and from those with wild type expression of STS (JEG-3), using an antiserum dilution of 1:250 (Fig. 2B). STS expression was detected in MCF-7 and JEG-3 cells but little expression was detected in COS-1 cells.

\section{$N$-terminal and $C$-terminal truncation}

To investigate the role of the N-terminal and C-terminal regions on the enzyme activity, monkey kidney COS-1 cells, which show negligible STS activity (Stein et al., 1989), and ER positive human breast cancer MCF-7 cells were transiently transfected with pSTSwt, pSTS1-184 (which lacks the C-terminal region, Fig. 1B) and with pSTS21-583 (which lacks the N-terminal signal sequence, Fig. 1C). The non-transfected MCF-7wt cells had an STS activity 10 times higher than the non-transfected COS-1wt cells. COS-1 cells transfected with the pSTSwt vector had a 26.5 fold increase in STS activity compared to mock-transfected COS-1 cells (Fig. 3A). In COS-1 cells transfected with pSTS1-184 or pSTS21-583, the STS activity was significantly lower than the STS activity in COS-1 cells transfected with wild type STS cDNA, and was not significantly different from mock-transfected cells.

There was a 14-fold increase in STS activity when MCF-7 cells were transfected with pSTSwt 
compared to mock-transfected cells (Fig. 3B). However, STS activity was 10-fold lower when compared to COS-1 cells transfected with pSTSwt. The STS activity level in MCF-7 cells transfected with pSTS1-184 or pSTS21-583 was significantly lower than that in MCF-7 cells transfected with pSTSwt, and was comparable to the STS activity of mock-transfected cells. To examine expression of the truncated STS proteins, the total protein extracts from COS-1 cells and MCF-7 cells transfected with the truncated STS expression vectors were analysed by immunoblotting with the anti-human STS antibody. The immunoblot showed a band at $61 \mathrm{kDa}$ for the cells transfected with pSTSwt, but the anti-human STS antibody failed to detect the N- or C-terminal truncated STS proteins in either cell line (Fig. 3C, 3D). An increase in STS mRNA expression was detected by PCR following 72h transfection with pSTS1-184 and pSTS21-583 (data not shown), indicating these constructs were successfully transfected and transcribed.

\section{Point mutations}

To determine the effect of specific amino acids on the STS enzyme activity, point mutations were introduced into the full length STS cDNA sequence. COS-1 cells were transfected with four expression vectors containing STS cDNA in which proline 212 was replaced by a glycine, and histidine 136 was replaced by a tyrosine, a tryptophan or a phenylalanine (Fig. 1A). The STS activity in COS-1 cells transfected with pSTS-P212G was equal to that in COS-1 cells transfected with pSTSwt (Fig. 3E). STS activity in COS-1 cells transfected with pSTS-H136Y, pSTS-H136W and pSTS-H136F was significantly lower than that in COS-1 cells transfected with pSTSwt or pSTS-P212G, and was similar to the STS activity of mock-transfected COS-1 cells. Corresponding results were found in MCF-7 cells (Fig. 3F).

To ensure that the STS constructs containing the amino acid substitutions were being expressed and translated correctly, lysates were prepared from transfected COS-1 and MCF-7 cells and analysed by immunoblotting with the anti-human STS antibody. In both cell lines, STS proteins with single point mutations were detected at $61 \mathrm{kDa}$ and the steady state levels of the transfected STS mutant proteins were similar to the transfected STSwt protein levels (Fig. 3H). 


\section{Glycosylation}

As Stein et al. (Stein et al., 1989) indicated that only asparagine 47 and 259 are glycosylated in STS protein, COS-1 cells were transfected with pSTS-N47Q, pSTS-N259Q and with the double mutant pSTS-N47/259Q. When one or both of these glycosylation sites was mutated, the STS activity was significantly reduced and was comparable to STS activity of mock-transfected COS1 cells (Fig. 4A). Similar results were seen in MCF-7 cells (Fig. 4B).

To investigate STS protein expression and the glycosylation state of N47 and / or N259 mutants, COS-1 cells and MCF-7 cells were transfected, harvested and lysed, and the lysates were analysed by SDS-PAGE and immunoblotting with the anti-human STS antibody (Fig. 4C, 4E). An aliquot of each sample was deglycosylated and analyzed by immunoblotting (Fig. 4D). The different degree of glycosylation can be visualized by changes in the protein migration through the gel. The pSTS-N47/259Q migrated with a faster mobility than the pSTSwt protein. When the pSTS-N47/259Q was deglycosylated, it migrated even faster (Fig. 4D, lane 7 and 8), suggesting the existence of further glycosylation site(s) in addition to N47 and N259.

STS protein sequence analysis reveals four potential N-linked oligosaccharide glycosylation sites (Stein et al., 1989). Therefore, the other two other potential sites (N333 and N459) and a nonglycosylated site (N54) were mutated and cloned into the expression vector and transfected into COS-1 and MCF-7 cells. STS activities in COS-1 cells transfected with N333, N459 and N54 mutants were as high as the STS activity level in COS-1 cells transfected with pSTSwt (Fig. 4F). Similar results were found in MCF-7 cells (Fig. 4G).

To investigate whether these sites may still be glycosylated, despite playing no role in modulating STS enzyme activity, total protein extracts from COS-1 cells and MCF-7 cells transfected with N54Q, N333Q and N459Q were analyzed by immunoblotting. PSTS-N54Q (lane 4) ran with the same mobility as pSTSwt (lane 1), whereas pSTS-N333Q and pSTS-N459Q (lanes 2 and 4) ran with a slightly faster mobility (58-59 kDa) than pSTSwt (Fig. 4H, 4I), suggesting the loss of an oligosaccharide. To ensure that this difference in size was not an artifact, the experiment was repeated several times $(n=4)$. When the lysates were deglycosylated prior to immunoblotting all constructs ran with the same mobility (Fig. 4D). 


\section{Discussion}

The aim of these studies was to identify key amino acids involved in regulating the activity of STS, a key enzyme in the synthesis of active steroids. Mutations were introduced both into the proposed active site and into the four potential glycosylation sites within the amino acid sequence. Furthermore, the role of both the N-terminal signal sequence and C-terminal region were investigated using truncated STS cDNA sequences. Monkey kidney COS-1 cells were chosen because they have virtually no STS activity and are easily transfected with a high efficiency. The human breast cancer MCF-7 cell line was used because it is hormone-dependent, has STS enzyme activity and is of human origin, which should ensure the expressed STS enzyme is post-translationally processed correctly.

The putative N-terminal signal peptide of 21 or 24 amino acids contains the residues serine 21histidine 22 and alanine 23 - alanine 24 which can equally be recognized by signal peptidase (Heijne, 1986; Stein et al., 1989). Negligible STS activities were found when the N-terminal signal sequence was deleted both in monkey kidney COS-1 cells and MCF-7 breast carcinoma cells. The signal sequence deleted STS protein was not detected by immunoblot, although mRNA was detected by PCR, indicating the construct had been successfully transcribed. The Nterminal hydrophobic domain is known to be important for P450c21 protein stability (Hsu et al., 1993), and is involved in protein folding. The N-terminal truncated STS protein may not be correctly folded, increasing the rate of degradation, since denatured proteins are degraded faster than native protein forms (Pakula and Sauer, 1989). Alternatively, changes in the STS conformation may alter the antibody recognition site, preventing the STS antibody from binding to the protein.

The catalytic domain is proposed to be primarily located in the N-terminal region of the STS peptide, centred around histidine 136. Therefore a construct containing only the first $184 \mathrm{~N}$ terminal amino acids was engineered (pSTS1-184). A lack of the whole C-terminal region leads to an inactive STS cDNA construct in both COS-1 cells and MCF-7 cells. This protein lacks residues 468-500 and 548-568, which constitute the entrance to the active site (HernandezGuzman et al., 2003) and this may explain the lack of activity observed after transfection with this construct. The key role of the C-terminal region is supported by the observation that most of 
the point mutations related to XLI are found in the C-terminal region, between the two hydrophobic regions and after the second N-linked glycosylation site (N259) (Alperin and Shapiro, 1997). The C-terminal deleted STS protein fails to be recognized by the anti-human STS antibody, probably due to the length of this protein or to its rapid degradation. Histidine 136 is catalytically important as it is positively charged and localised inside the active site cavity. With four other positively charged side chains (arginine 79, lysine 134, histidine 290 and lysine 368) histidine 136 neutralizes the inside of the active site cavity and participates in the deprotonation of the hydroxyl on the sulphur atom (Hernandez-Guzman et al., 2003). COS-1 cells and MCF-7 cells transfected with STS cDNA constructs with point mutations at histidine 136 lack STS enzyme activity, despite expressing a protein of the same size and amount as pSTSwt-transfected cells. These results confirm the key role that histidine 136 plays in the enzyme activity of the STS protein. Using amino acid modification reagents, Purohit et al. (Purohit et al., 1995a) have previously reported that photo-oxidation of histidine with Rose Bengal decreased STS activity in a concentration-dependent manner.

The STS polypeptide contains two hydrophobic domains, which constitute a $\alpha$-helical structure (residues 185-237) and a $\beta$-sheet (residues 466-495). The first structure is interrupted by a turninducer, proline 212, which divides the hydrophobic sequence into two parts of 27 and 25 residues, each of sufficient length to span the membrane (Hernandez-Guzman et al., 2003). The replacement of the proline with a less flexible glutamine at amino acid 212 does not affect the STS activity, suggesting that a change in the STS conformation at this turning point does not affect the catalytic core of the enzyme. Immunoblot studies demonstrated that the P212Q mutant protein had the same steady state level of STS expression as the transfected STS wild type protein, and furthermore, that the mutation did not affect the antibody recognition of the STS protein. In contrast, removal of the gene sequence encoding the putative transmembrane domain of human placental STS and replacement with a Gly Gly Gly Gly Ser to act as a hinge did not result in a 'soluble' active form of the enzyme (Purohit et al., 1998).

Proteins acquire their secondary and tertiary structure in the lumen of the endoplasmic reticulum in mammals, where they are glycosylated. The N-linked oligosaccharide sites are essential for the folding of glycoproteins (Hurtley and Helenius, 1989). Two (asparagine 47 and asparagine 259) of the four potential N-glycosylation sites of STS are thought to be glycosylated (Stein et al., 1989). When N47 and / or N259 are mutated, the STS enzyme is inactive. Immunoblot 
studies revealed the presence of proteins carrying these mutations at a steady state level comparable to the transfected wild type protein expression level. These findings agree with Stein et al. (Stein et al., 1989) whose studies showed the importance of the N47 and N259 glycosylation sites of STS. However, analysis of the immunoblot showed that the double mutant pSTS-N47/259Q protein has a faster mobility on an SDS-PAGE gel than pSTSwt protein, and that it acquires a higher mobility after deglycosylation, suggesting that other N-linked oligosaccharide sites are used for glycosylation.

Studies of pSTS-N333Q and pSTS-N459Q showed that these mutations do not affect STS activity or protein expression. Furthermore, immunoblots analysis showed that the N333Q and N459Q mutant proteins run faster than STS wild type protein, suggesting that asparagine 333 and asparagine 459 are used for N-linked glycosylation but do not affect the sulphatase activity. In conclusion, we have shown that the N-terminal signal sequence is important for STS enzyme activity as well as the C-terminal region. These data have also shown that histidine 136, responsible for the deprotonation of the sulphate moiety's group during catalysis (HernandezGuzman et al., 2003), is essential for STS enzyme activity. Moreover, a mutation in proline 212, which links the two hydrophobic parts and allows the turn into the membrane, does not affect the STS enzyme activity. Finally, we have shown that the four potential N-linked oligosaccharide sites are glycosylated, but only asparagine 47 and asparagine 259 are important for STS enzyme activity.

These studies aid the understanding of how the new anti-cancer drug target, STS (Stanway et al., 2006), catalyses the conversion of inactive steroids, such as E1S, to active estrogens and may support further rational drug design. Indeed, several mechanisms of action for a sulphamatebased active site-directed steroid sulphatase inhibitor were proposed and sulphamates esters have been developed (Woo et al., 2000). One of them, 667COUMATE, an orally active, non-steroidal, non-estrogenic and highly potent active site-directed STS inhibitor, is in pre-clinical development and showed very encouraging results during the Phase I clinical trial (Stanway et al., 2006). In the future, based on a better knowledge of STS enzyme, even more powerful STS inhibitor than 667COUMATE will be developed and their utilisation for other pathologies will be investigated. 


\section{References}

Alperin, E.S., and Shapiro, L.J. (1997). Characterization of point mutations in patients with Xlinked ichthyosis. Effects on the structure and function of the steroid sulfatase protein. J Biol Chem 272, 20756-20763.

Basler, E., Grompe, M., Parenti, G., Yates, J., and Ballabio, A. (1992). Identification of point mutations in the steroid sulfatase gene of three patients with X-linked ichthyosis. Am J Hum Genet 50, 483-491.

Billich, A., Nussbaumer, P., and Lehr, P. (2000). Stimulation of MCF-7 breast cancer cell proliferation by estrone sulfate and dehydroepiandrosterone sulfate: inhibition by novel nonsteroidal steroid sulfatase inhibitors. J Steroid Biochem Mol Biol 73, 225-235.

Conary, J., Nauerth, A., Burns, G., Hasilik, A., and von Figura, K. (1986). Steroid sulfatase. Biosynthesis and processing in normal and mutant fibroblasts. Eur J Biochem 158, 71-76.

Elger, W., Schwarz, S., Hedden, A., Reddersen, G., and Schneider, B. (1995). Sulfamates of various estrogens are prodrugs with increased systemic and reduced hepatic estrogenicity at oral application. J Steroid Biochem Mol Biol 55, 395-403.

Engvall, E., and Perlman, P. (1971). Enzyme-linked immunosorbent assay (ELISA). Quantitative assay of immunoglobulin G. Immunochemistry 8, 871-874.

Gonzalez-Huerta, L.M., Riviera-Vega, M.R., Kofman-Alfeuro, S.H., and Cuevas-Covarrubias, S.A. (2003). Novel missense mutation (Arg432Cys) in a patient with steroid sulphatasedeficiency. Clin Endocrinol (Oxf) 59, 263-264.

Heijne, G.V. (1986). The distribution of positively charged residues in bacterial inner membrane proteins correlates with the trans-membrane topology. Embo J 5, 3021-3027.

Hernandez-Guzman, F.G., Higashiyama, T., Pangborn, W., Osawa, Y., and Ghosh, D. (2003). Structure of human estrone sulfatase suggests functional roles of membrane association. J Biol Chem 278, 22989-22997.

Hsu, L.C., Hu, M.C., Cheng, H.C., Lu, J.C., and Chung, B.C. (1993). The N-terminal hydrophobic domain of $\mathrm{P} 450 \mathrm{c} 21$ is required for membrane insertion and enzyme stability. J Biol Chem 268, 14682-14686.

Hurtley, S.M., and Helenius, A. (1989). Protein oligomerization in the endoplasmic reticulum. Annu Rev Cell Biol 5, 277-307.

Miyoshi, Y., Ando, A., Hasegawa, S., Ishitobi, M., Taguchi, T., Tamaki, Y., and Noguchi, S. (2003). High expression of steroid sulfatase mRNA predicts poor prognosis in patients with estrogen receptor-positive breast cancer. Clin Cancer Res 9, 2288-2293.

Pakula, A.A., and Sauer, R.T. (1989). Genetic analysis of protein stability and function. Annu Rev Genet 23, 289-310.

Pasqualini, J.R., Ebert, C., and Chetrite, G.S. (2001). Biological effects of progestins in breast cancer. Gynecol Endocrinol 15 Suppl 6, 44-52.

Purohit, A., Hejaz, H.A., Woo, L.W., van Strien, A.E., Potter, B.V., and Reed, M.J. (1999). Recent advances in the development of steroid sulphatase inhibitors. J Steroid Biochem Mol Biol 69, 227-238.

Purohit, A., Potter, B.V., Parker, M.G., and Reed, M.J. (1998). Steroid sulphatase: expression, isolation and inhibition for active-site identification studies. Chem Biol Interact 109, 183-193. Purohit, A., Williams, G.J., Howarth, N.M., Potter, B.V., and Reed, M.J. (1995a). Inactivation of steroid sulfatase by an active site-directed inhibitor, estrone-3-O-sulfamate. Biochemistry 34, 11508-11514. 
Purohit, A., Williams, G.J., Roberts, C.J., Potter, B.V., and Reed, M.J. (1995b). In vivo inhibition of oestrone sulphatase and dehydroepiandrosterone sulphatase by oestrone-3-Osulphamate. Int J Cancer 63, 106-111.

Reed, M.J., Purohit, A., Woo, L.W., Newman, S.P., and Potter, B.V. (2005). Steroid sulfatase: molecular biology, regulation, and inhibition. Endocr Rev 26, 171-202.

Shapiro, L.J., Yen, P., Pomerantz, D., Martin, E., Rolewic, L., and Mohandas, T. (1989). Molecular studies of deletions at the human steroid sulfatase locus. Proc Natl Acad Sci U S A $86,8477-8481$.

Stanway, S.J., Purohit, A., Woo, L.W., Sufi, S., Vigushin, D., Ward, R., Wilson, R.H., Stanczyk, F.Z., Dobbs, N., Kulinskaya, E., et al. (2006). Phase I study of STX 64 (667 Coumate) in breast cancer patients: the first study of a steroid sulfatase inhibitor. Clin Cancer Res 12, 1585-1592.

Stein, C., Hille, A., Seidel, J., Rijnbout, S., Waheed, A., Schmidt, B., Geuze, H., and von Figura, K. (1989). Cloning and expression of human steroid-sulfatase. Membrane topology, glycosylation, and subcellular distribution in BHK-21 cells. J Biol Chem 264, 13865-13872.

Sugawara, T., Nomura, E., and Hoshi, N. (2006). Both N-terminal and C-terminal regions of steroid sulfatase are important for enzyme activity. J Endocrinol 188, 365-374.

Sugawara, T., Shimizu, H., Hoshi, N., Fujimoto, Y., Nakajima, A., and Fujimoto, S. (2000). PCR diagnosis of X-linked ichthyosis: identification of a novel mutation (E560P) of the steroid sulfatase gene. Hum Mutat 15, 296.

Utsumi, T., Yoshimura, N., Maruta, M., Takeuchi, S., Ando, J., Maeda, K., and Harada, N. (1999). Significance of Steroid Sulfatase Expression in Human Breast Cancer. Breast Cancer 6, 298-300.

Valdes-Flores, M., Jimenez Vaca, A.L., Kofman-Alfaro, S.H., and Cuevas-Covarrubias, S.A. (2001). Characterization of a novel point mutation (Arg432His) in X-linked ichthyosis. Acta Derm Venereol 81, 54-55.

von Figura, K., Schmidt, B., Selmer, T., and Dierks, T. (1998). A novel protein modification generating an aldehyde group in sulfatases: its role in catalysis and disease. Bioessays 20, 505510.

Warren, J.C., and French, A.P. (1965). Distribution of Steroid Sulfatase in Human Tissues. J Clin Endocrinol Metab 25, 278-282.

Woo, L.L., Purohit, A., Malini, B., Reed, M.J., and Potter, B.V. (2000). Potent active sitedirected inhibition of steroid sulphatase by tricyclic coumarin-based sulphamates. Chem Biol 7, 773-791.

Yen, P.H., Allen, E., Marsh, B., Mohandas, T., Wang, N., Taggart, R.T., and Shapiro, L.J. (1987). Cloning and expression of steroid sulfatase cDNA and the frequent occurrence of deletions in STS deficiency: implications for X-Y interchange. Cell 49, 443-454. 


\section{Legends}

Figure 1. Structure of the STS polypeptide and of the truncated STS. The black boxes represent the 22 amino acids signal sequence. The dotted boxes represent the N-terminal domain. The striped boxes represent the hydrophobic transmembrane domain. The squared boxes represent the $\mathrm{C}$-terminal domain. The predicted glycosylation sites are indicated by $\xi$. The relative locations of the point mutations proline 212 and histidine 136 and their amino acids substitutions are shown by $\uparrow$. A: complete sequence (pSTSwt); B: C-terminally truncated STS (pSTS1-184); C: N-terminal signal sequence truncated STS (pSTS21-583).

Figure 2: Characterisation of the anti-human STS antisera. (A) The immunoreactivity of antisera raised against human recombinant STS in three mice and two rabbits was assayed by ELISA. All antisera recognised the STS-His 6 protein, with the two rabbit antisera having the strongest immunoreactivity. Anti-His6: monoclonal antibody to the His 6 tag, SKM13, 14 \& 15: antisera raised in mice, SK3706 \& 3707: antisera raised in rabbits. (B) Immunoblot analysis of STS expression in cell lysates (20 $\mu \mathrm{g}$ protein) using the rabbit anti-human antiserum SK3706 at a dilution of 1:250. 1: MCF-7, 2: JEG-3, 3: 293-EBNA[his $\left.{ }_{6} S T S\right]$, 4: COS-1, 5: COS-1.STS, 6: MagicMark protein standard (Invitrogen).

Figure 3. STS truncated and STS point mutations: enzyme activity and protein expression in COS-1 cells and MCF-7 cells. COS-1 and MCF-7 cells were transiently transfected with pSTS1-184, pSTS21-583, pSTS-P212G, pSTS-H136Y, pSTS-H136W and pSTS-H136F expression vectors. STS activity was assayed in COS-1 cells (A, E) and in MCF-7 cells (B, F). Three independent assays were performed in duplicate and the results presented are the average of activities relative to activity in mock-transfected cells (100\%), bars, SE. The STS protein expression was examined by immunoblotting with an anti-human STS antibody. STS truncated protein was detected at approximately $61 \mathrm{kDa}$ for COS-1 cells (C) and MCF-7 cells (D); 1: Mock, 2: pSTSwt, 3: pSTS1-184, 4: pSTS21-583. STS point mutation protein was detected at approximately $61 \mathrm{kDa}$ for COS-1 cells $(\mathrm{G})$ and MCF-7 cells (H); 1: Mock, 2: pSTSwt, 3: pSTSP212G, 4: pSTS-H136Y, 5: pSTS-H136W, 6: pSTS-H136F. 
Figure 4. STS glycosylation sites mutations: enzyme activity and protein expression in COS-1 cells and MCF-7 cells. COS- 1 and MCF-7 cells were transiently transfected with pSTSN47Q, pSTS-N259Q, pSTS-N47/259Q, pSTS-N333Q and pSTS-N459Q expression vectors. STS activity was assayed in COS-1 cells (A, F) and in MCF-7 cells (B, G). Three independent assays were performed in duplicate and the results presented are the average of activities relative to activity in mock-transfected cells $(100 \%)$, bars, SE. STS protein expression was examined by immunoblotting with an anti-human STS antibody. The STS protein mutated in N47 and/or N259 was detected at approximately $61 \mathrm{kDa}$ for COS-1 cells (C) and MCF-7 cells (E). 1: Mock, 2: pSTSwt, 3: pSTS-N47Q, 4: pSTS-N259Q, 5: pSTS-N47/259Q. An aliquot of each sample was deglycosylated (DG) and analysed by SDS-PAGE (D). 1: pSTSwt, 2: pSTSwt DG, 3: pSTSN259Q, 4: pSTS-N259Q DG, 5: pSTS-N47Q, 6: pSTS-N47Q DG, 7: pSTS-N47/259Q, 8: pSTSN47/259Q DG. STS protein mutated in N333 or N459 was detected at about $61 \mathrm{kDa}$ for COS-1 cells (H) and MCF-7 cells (I). 1: pSTSwt, 2: pSTS-N333Q, 3: pSTS-N459Q, 4: pSTS-N54Q. 


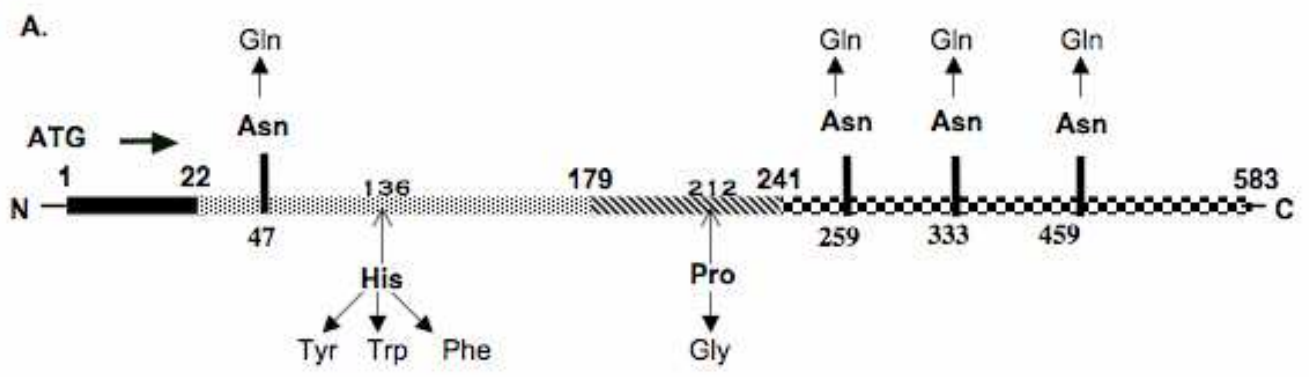

B.

ATG $\rightarrow$

$\mathrm{N}-1$

c. ATG -

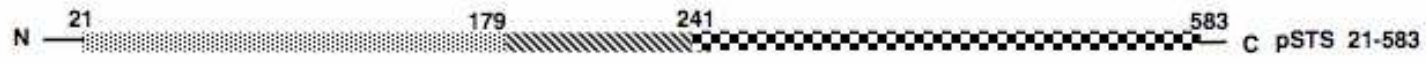


A.

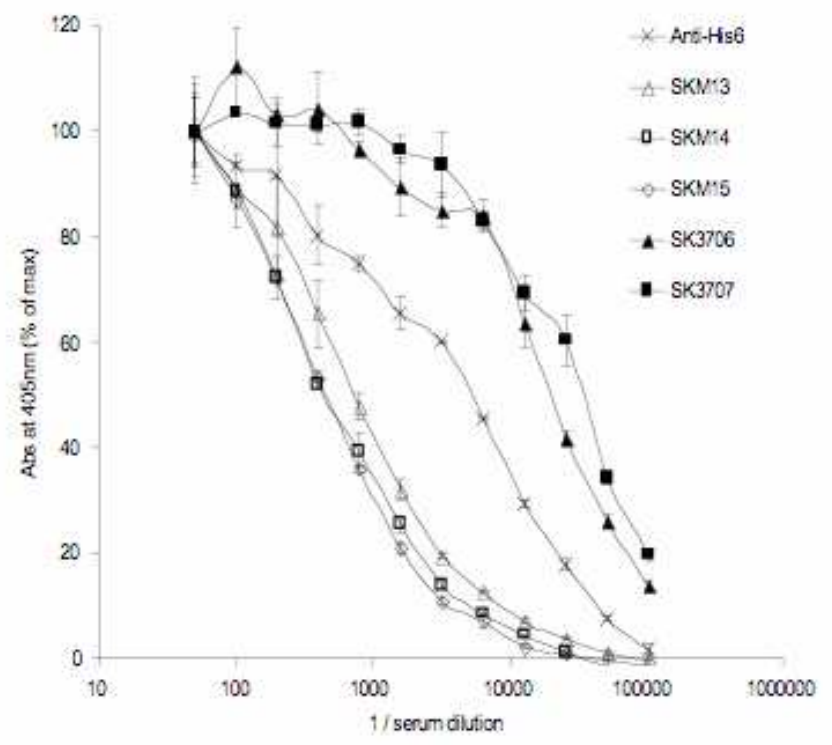

B.

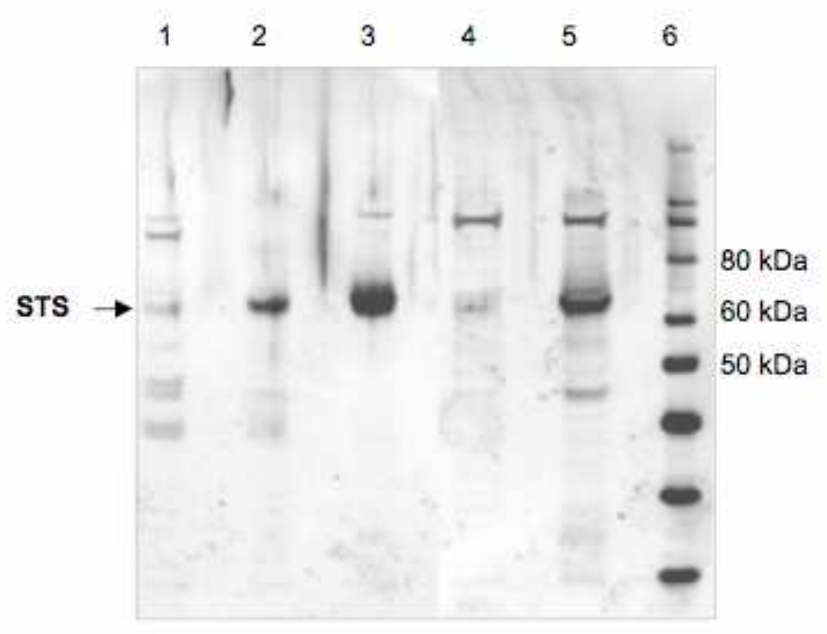



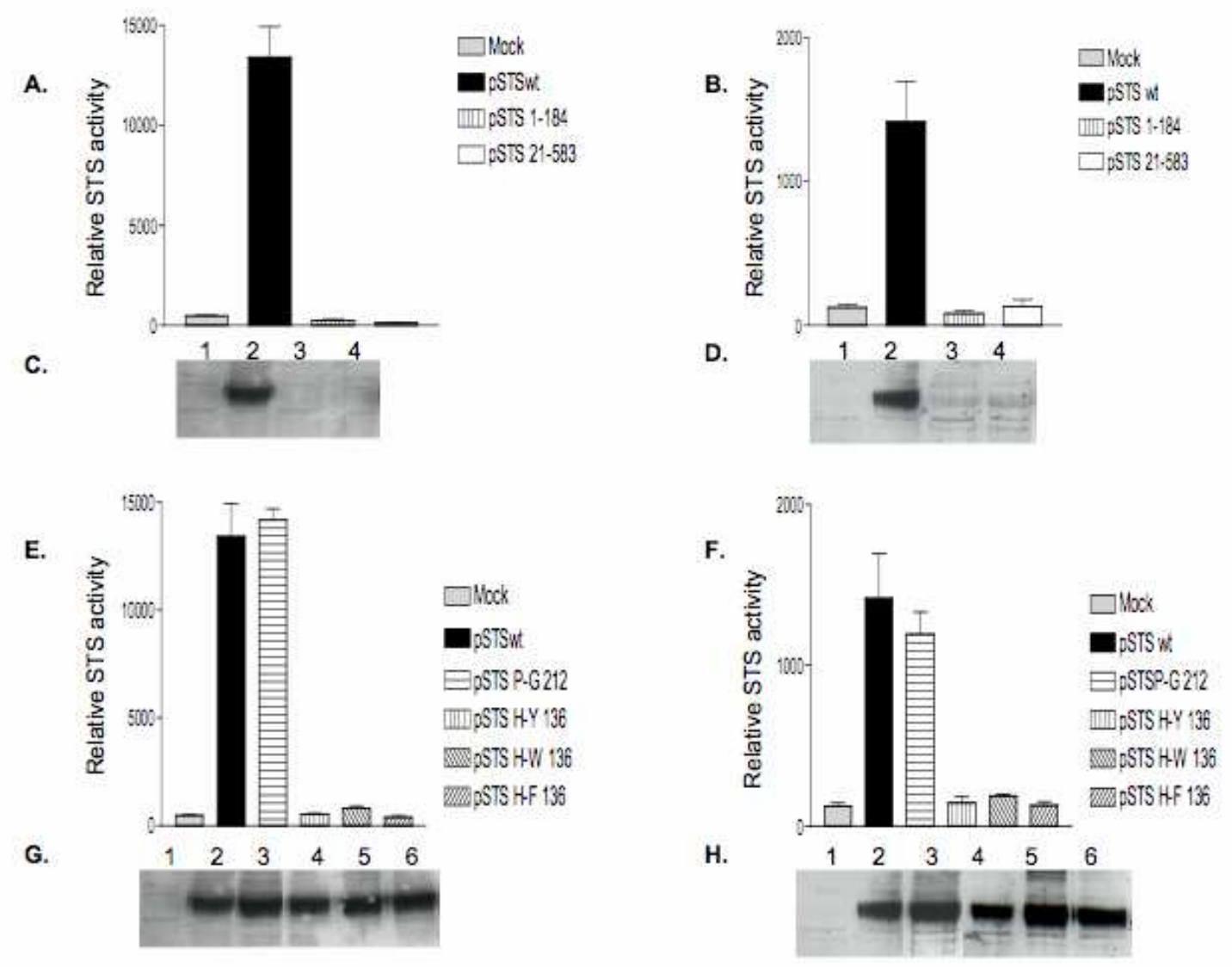
A.

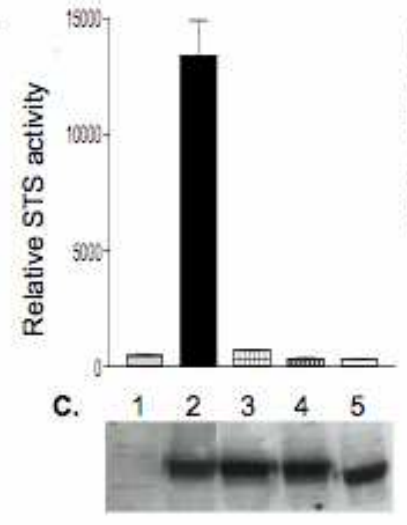

F.

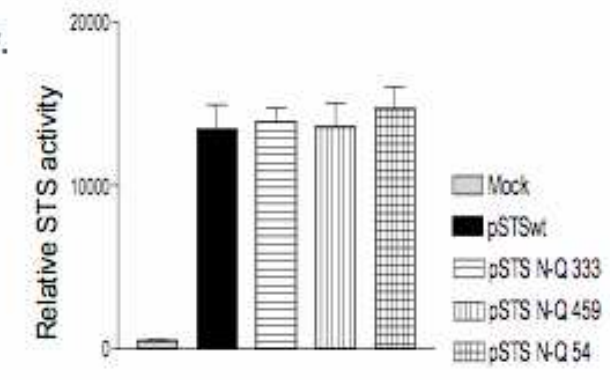

H.

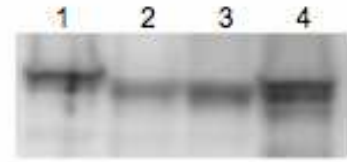

B.
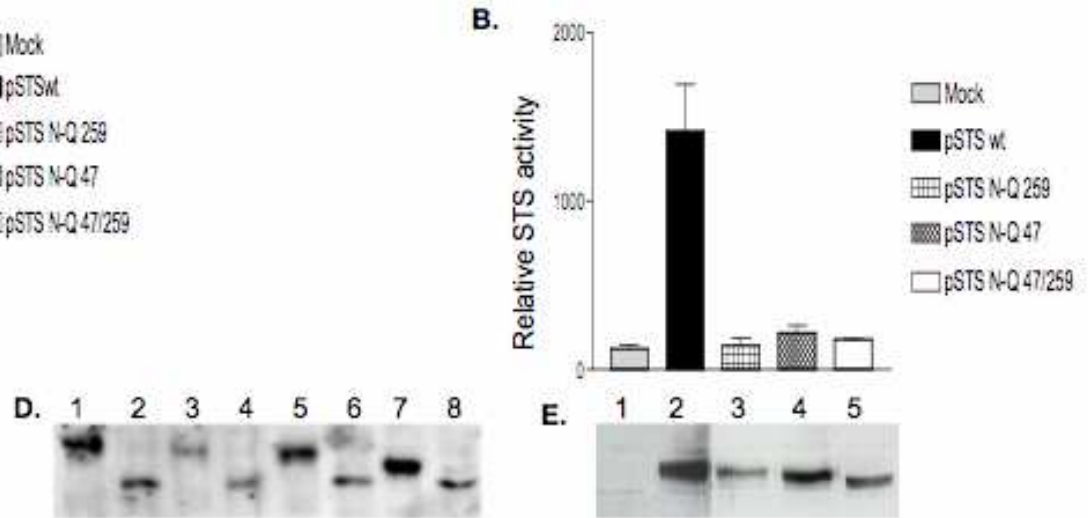

G.

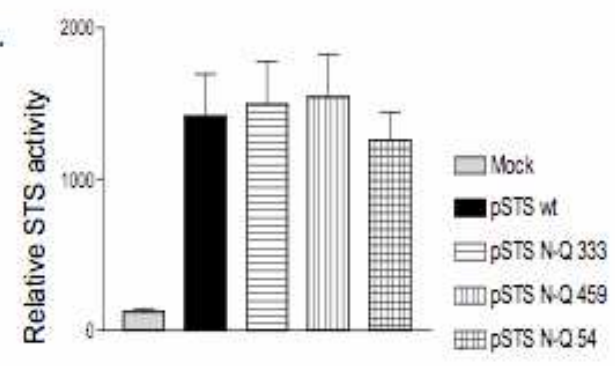

I.

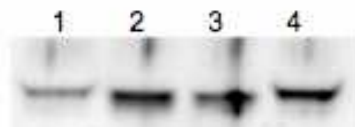

